Article

\title{
Hysteresis in Lanthanide Aluminum Oxides Observed by Fast Pulse CV Measurement
}

\section{Chun Zhao ${ }^{1, \dagger}$, Ce Zhou Zhao ${ }^{1,2, *}$, Qifeng Lu ${ }^{1}$, Xiaoyi Yan ${ }^{1,2}$, Stephen Taylor ${ }^{1}$ and}

Paul R. Chalker ${ }^{3}$

1 Department of Electrical Engineering and Electronics, University of Liverpool, Liverpool L69 3GJ, UK; E-Mails: chun.zhao@liverpool.ac.uk (C.Z.); qifeng@liverpool.ac.uk (Q.L.); xiaoyi.yan10@student.xjtlu.edu.cn (X.Y.); s.taylor@liverpool.ac.uk (S.T.)

2 Department of Electrical and Electronic Engineering, Xi'an Jiaotong-Liverpool University, Suzhou 215123, China

3 Department of Materials Science and Engineering, University of Liverpool, Liverpool L69 3GH, UK; E-Mail: pchalker@liverpool.ac.uk

$\dagger$ Present address: Nano \& Advanced Materials Institute, Hong Kong University of Science and Technology, Hong Kong; E-Mail: garyzhao@ust.hk.

* Author to whom correspondence should be addressed; E-Mail: cezhou.zhao@xjtlu.edu.cn or cezhou@liverpool.ac.uk; Tel.: +86-512-8816-1408.

External Editor: Durga Misra

Received: 15 January 2014; in revised form: 29 September 2014 / Accepted: 8 October 2014 / Published: 13 October 2014

\begin{abstract}
Oxide materials with large dielectric constants (so-called high- $k$ dielectrics) have attracted much attention due to their potential use as gate dielectrics in Metal Oxide Semiconductor Field Effect Transistors (MOSFETs). A novel characterization (pulse capacitance-voltage) method was proposed in detail. The pulse capacitance-voltage technique was employed to characterize oxide traps of high- $k$ dielectrics based on the Metal Oxide Semiconductor (MOS) capacitor structure. The variation of flat-band voltages of the MOS structure was observed and discussed accordingly. Some interesting trapping/detrapping results related to the lanthanide aluminum oxide traps were identified for possible application in Flash memory technology. After understanding the trapping/detrapping mechanism of the high- $k$ oxides, a solid foundation was prepared for further exploration into charge-trapping non-volatile memory in the future.
\end{abstract}


Keywords: high- $k$ dielectrics; lanthanide aluminum oxides; pulse capacitance-voltage (CV); oxide traps

\section{Introduction}

If $\mathrm{SiO}_{2}$ gate dielectric thickness is reduced below $1.4 \mathrm{~nm}$, the resulting high gate leakage current levels due to electron tunneling effects become unacceptable for device reliability [1,2]. The decreasing sizes in complementary metal oxide semiconductor (CMOS) transistor technology required the replacement of $\mathrm{SiO}_{2}$ with gate dielectrics that have a high dielectric constant (high- $k$ ) $[3,4]$. The equivalent oxide thickness (EOT) is the thickness of $\mathrm{SiO}_{2}$ gate oxide needed to obtain the same gate capacitance as that obtained with thicker high- $k$ dielectrics. Thicker equivalent oxide thickness, to reduce the leakage current of gate oxides, was obtained by introducing the high- $k$ dielectric into real applications from $45 \mathrm{~nm}$ node technology. In recent years, various high- $k$ gate dielectrics have been investigated to find suitable alternative materials [5-12]. Significant progress has been made on the screening and selection of high- $k$ gate dielectrics, understanding their physical properties, and their integration into CMOS technology [13-20]. Now it is recognized that a large family of oxide-based materials emerges as candidates to replace $\mathrm{SiO}_{2}$ gate dielectrics in advanced CMOS applications [21-26]. Among them are cerium oxide $\mathrm{CeO}_{2}$ [27], cerium zirconate $\mathrm{CeZrO}_{4}$ [28], gadolinium oxide $\mathrm{Gd}_{2} \mathrm{O}_{3}$ [29], erbium oxide $\mathrm{Er}_{2} \mathrm{O}_{3}$ [30], neodymium oxide $\mathrm{Nd}_{2} \mathrm{O}_{3}$ [31], aluminum oxide $\mathrm{Al}_{2} \mathrm{O}_{3}$ [32], lanthanum aluminum oxide $\mathrm{LaAlO}_{3}$ [33], lanthanum oxide $\mathrm{La}_{2} \mathrm{O}_{3}$ [34], yttrium oxide $\mathrm{Y}_{2} \mathrm{O}_{3}$ [35], tantalum pentoxide $\mathrm{Ta}_{2} \mathrm{O}_{5}$ [36], titanium dioxide $\mathrm{TiO}_{2}$ [37], zirconium dioxide $\mathrm{ZrO}_{2}$ [38], lanthanum doped zirconium oxide $\mathrm{La}_{x} \mathrm{Zr}_{1-x} \mathrm{O}_{2-\delta}$ [39], hafnium oxide $\mathrm{HfO}_{2}$ [40], $\mathrm{HfO}_{2}$-based oxides $\mathrm{La}_{2} \mathrm{Hf}_{2} \mathrm{O}_{7}$ [41], $\mathrm{Ce}_{x} \mathrm{Hf}_{1-x} \mathrm{O}_{2}$ [42], hafnium silicate $\mathrm{HfSi}_{x} \mathrm{O}_{y}$ [43], and rare-earth scandates $\mathrm{LaScO}_{3}$ [44], $\mathrm{GdScO}_{3}$ [45], $\mathrm{DyScO}_{3}$ [46], and $\mathrm{SmScO}_{3}$ [47]. Excellent device results have been obtained using $\mathrm{La}_{2} \mathrm{O}_{3}$ gate dielectric layers $(k \sim 27)$, with a $3.3 \mathrm{~nm}$ thick $\mathrm{La}_{2} \mathrm{O}_{3}$ layer giving a $\mathrm{SiO}_{2} \mathrm{EOT}$ as low as $0.48 \mathrm{~nm}$, together with very low leakage currents [48]. However, $\mathrm{La}_{2} \mathrm{O}_{3}$ is chemically unstable and is easily converted to $\mathrm{La}(\mathrm{OH})_{3}$ on reaction with ambient water. $\mathrm{LaAlO}_{3}$ combines the advantages of the high permittivity of $\mathrm{La}_{2} \mathrm{O}_{3}$ with the chemical and thermal stability of $\mathrm{Al}_{2} \mathrm{O}_{3} . \mathrm{LaAlO}_{3}$ has a permittivity of 22-27, a large band gap $(6.2 \mathrm{eV})$, and high band offsets on $\mathrm{Si}$ (1.8 eV for electrons and $3.2 \mathrm{eV}$ for holes). Equivalent $\mathrm{SiO}_{2}$ thicknesses as low as 0.9-1.1 nm have therefore been achieved using $\mathrm{LaAlO}_{3}$. Recently, to enhance the thermal stability is the incorporation of aluminum (Al) to develop innovative multifunctional advanced lanthanide aluminates-based ceramics, $\mathrm{NdAlO}_{3}$ [49]. They remain amorphous up to high temperatures, leading to a large reduction in leakage current relative to poly-crystalline $\mathrm{Nd}_{2} \mathrm{O}_{3}$ films during CMOS processing. Therefore, the lanthanide aluminates, $\mathrm{MAlO}_{3}(\mathrm{M} \sim \mathrm{La}, \mathrm{Nd})$, are promising high- $k$ materials for next generation CMOS applications.

The rapid growth of Flash memory technology has been motivated by the continuous downscaling of memory cells [50,51]. Starting from the advanced technology generation for charge-trapping Flash devices, the spacing between two adjacent gates became too narrow to arrange the metal gate to overlap the floating gate vertically in minimum feature-sized standard cells $[52,53]$. The introduction of high- $k$ materials into floating-gate Flash memory has been proposed as a potential solution [54-56]. 
A fundamental understanding of the trap mechanism in new dielectric materials was critical for write/erase, retention and endurance properties of Flash memory [57-59]. Therefore, new ideas and approaches are required. These include: Negative-Bias Temperature Instability (NBTI), NBTI lifetime prediction, fast reliability screening and charge pumping techniques. These measurement techniques are required to understand of the underlying science of these dielectrics. Reliability degradation, defect loss, slowdown, and device lifetime enhancement, energy and spatial distribution, electron trapping and interface states, time-dependent defect variation were actively investigated for the high- $k$ dielectrics for several years.

Prior to this paper, many novel measurement approaches were proposed for the next generation Flash memory since 2005 [60-68]. Various significant findings related to traps within the gate oxides were also reported [69-72]. In this paper, a novel electrical characterization of Metal Oxide Semiconductor (MOS) capacitance: pulse capacitance-voltage (CV), was proposed in detail. The accuracy and reliability of the pulse $\mathrm{CV}$ testing system was comprehensively verified and examined, and shown to be a powerful measurement method for dielectric characterization of high- $k$ materials. Large hysteresis effects caused by oxide traps were observed in lanthanide aluminum oxides, which was critical for the development of the charge-trapping Flash memory in the future.

\section{Experimental Section}

High- $k$ dielectrics, $\mathrm{LaAlO}_{3}, \mathrm{NdAlO}_{3}$ thin films, were deposited on $n$-type $\mathrm{Si}(100)$ substrates using liquid injection atomic layer deposition (ALD), carried out on an Aixtron AIX 200FE AVD reactor (Aixtron, Herzogenrath, Germany) fitted with the "Trijet"TM liquid injector system. The $\mathrm{LaAlO}_{3}$ thin films grown by ALD are all La deficient, with the La:Al ratio varying from 0.50 to 0.61 over the growth temperature range of $160-300{ }^{\circ} \mathrm{C}$. As gas phase reactions are absent in ALD, the precursor is likely to remain intact until reaching the growth surface. It is therefore unsurprising that there is little variation in the La:Al ratio, although the reason for the La deficiency in the thin films is not known. The thickness of the $\mathrm{LaAlO}_{3}$ thin film grown by ALD is $28 \mathrm{~nm}$. Near stoichiometric $\mathrm{NdAlO}_{3}$ thin films were grown by utilizing the single-source precursor $\left[\mathrm{NdAl}\left(\mathrm{OPr}^{i}\right)_{6}\left(\mathrm{Pr}_{i} \mathrm{OH}\right)\right]_{2}$. Selected thin films were subjected to high-temperature $\left(750-950{ }^{\circ} \mathrm{C}\right)$ post-deposition annealing (PDA) in pure nitrogen $\left(\mathrm{N}_{2}\right)$ ambient for $60 \mathrm{~s}$. Subsequently, a post-metallization forming gas anneal (FGA) was carried out at $400{ }^{\circ} \mathrm{C}$ for $30 \mathrm{~min}$ using $\mathrm{H}_{2}: \mathrm{N}_{2}$ in the ratio $1: 9$, together with a control as-deposited sample. The high- $k$ thickness and a thin native oxide interlayer, adjacent to the silicon substrate, changed from 11 and $1.5 \mathrm{~nm}$, respectively, to 10.4 and $2.5 \mathrm{~nm}$, respectively, after $950{ }^{\circ} \mathrm{C}$ PDA. This could be due to inter-diffusion of oxygen between $\mathrm{SiO}_{2}$ and $\mathrm{NdAlO}_{3}$. Here, $\mathrm{NdAlO}_{3}$ thin films samples discussed in the manuscript was after PDA. A thermal $\mathrm{SiO}_{2}$ sample was grown using dry oxidation at $1100{ }^{\circ} \mathrm{C}$ to provide a comparison with the high- $k$ stacks. MOS capacitors were fabricated by thermal evaporation of Au gates through a shadow mask with an effective area of $4.9 \times 10^{-4} \mathrm{~cm}^{2}$. The backside contact of selected Si wafers was cleaned with a buffer HF solution and subsequently a $200 \mathrm{~nm}$ thickness of Al film was deposited on it by thermal evaporation.

The pulse CV measurement system was developed and implemented to probe the MOS capacitor sample with high- $k$ thin film, and its system structure chart is shown in Figure 1. We used a functional/arbitrary waveform generator to input a pulse voltage waveform $\left(v_{g}\right)$ for the sample. The related current through the sample ( $\left.i_{\text {total }}\right)$ was fed into a current amplifier and then was amplified as an 
output voltage signal $\left(v_{\mathrm{CH}}\right)$. Channel two of the oscilloscope was used to track the output voltage, while the input pulse voltage waveform was monitored in channel one. There was no specific requirement for the oscilloscope model. Here, the DG1302 oscilloscope was employed. In terms of functional/arbitrary waveform generator, DG3061 (RIGOL, Beijing, China)/DG2041 (RIGOL)/HP8110 (Agilent, Santa Clara, CA, USA) could be implemented to produce pulse voltage. The Keithley 428 current amplifier (Keithley, Cleveland, OH, USA) was selected for the pulse CV measurement system. The symbol diagram of an under-testing device is also modeled in the inset of Figure 1. For MOS capacitor samples with the high- $k$ thin film, we could make it simple as the parallel combination of a capacitor (C) and a resistor (R). Accordingly, the current flowing through the capacitor and resistor are $i_{C}$ and $i_{R}$, respectively. Details are discussed in the following section.

Figure 1. Pulse capacitance-voltage (CV) measurement system structure chart. A functional/arbitrary waveform generator was implemented to generate the voltage pulse waveform. The current through the under-testing device was fed into a current amplifier and then was amplified as an output voltage signal. An oscilloscope was used to monitor the input and output voltage signals.

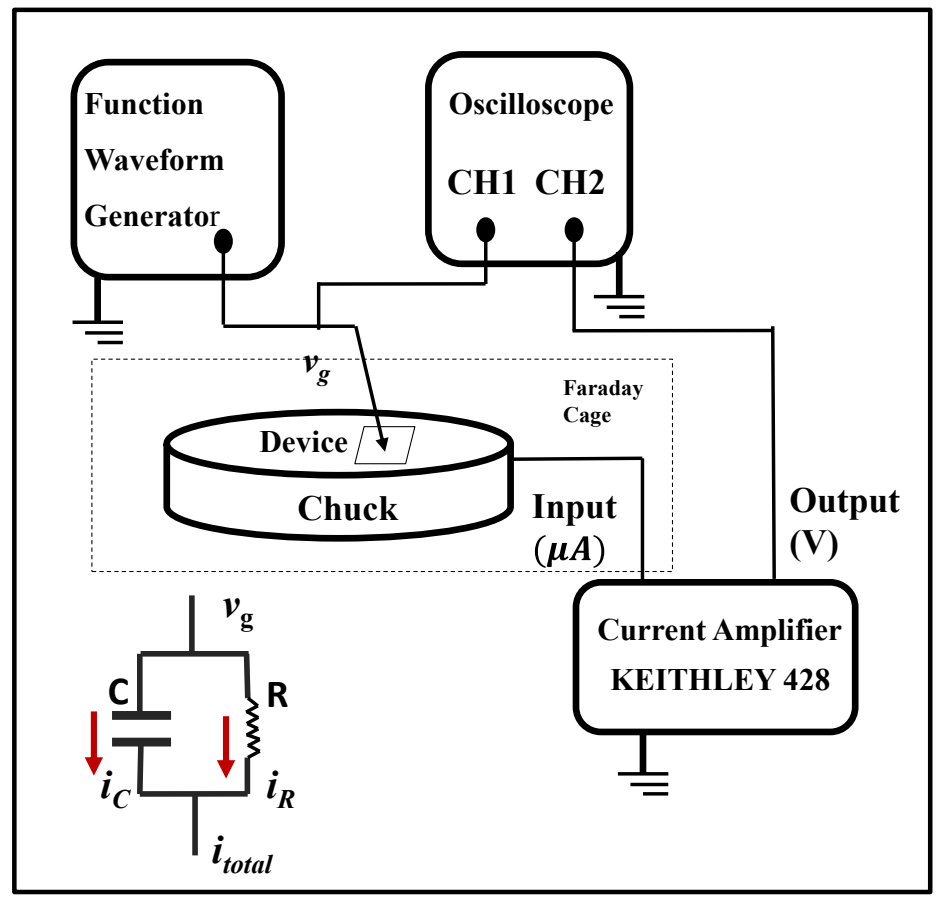

\section{Results and Discussion}

In order to verify the working principle behind the pulse CV measurement system, firstly, a discrete commercial ceramic capacitor with a near infinite resistance value was used to replace the MOS capacitor sample shown in Figure 1. The value of the capacitance component was below $1000 \mathrm{pF}$. Via the relation between voltage and current, the following formula is derived:

$$
i_{C}(t)=\frac{v_{\mathrm{CH} 2}(t)}{A}=C \cdot \frac{d v_{g}(t)}{d t}
$$


where $v_{\mathrm{CH} 2}$ is the output voltage of the current amplifier (recoded by channel two of the oscilloscope). $\nu_{g}$ is the input voltage of the sample. $A$ is an amplification factor of the current amplifier and $C$ is the capacitance value of the device under test. Re-arranging, $v_{\mathrm{CH} 2}$ could be obtained:

$$
v_{\mathrm{CH} 2}(t)=A \cdot C \cdot \frac{d v_{g}(t)}{d t}
$$

Based on the formula above, the theoretical $v_{\mathrm{CH} 2}$ could be calculated as a reference to compare the measurement results. In Figure 2a, the measurement results from channel one (green) and channel two (purple) of the oscilloscope are presented. Following the derived formula, we plotted the theory data for channel one (blue) and channel two (red). Obviously, it was observed that the theory data fitted well with measurement results.

Figure 2. (a) Voltage (V) versus time ( $\mu \mathrm{s})$ of a discrete capacitor. (b) Voltage (V) versus time $(\mu \mathrm{s})$ of a discrete resistor. (c) Voltage $(\mathrm{V})$ versus time $(\mu \mathrm{s})$ of a discrete capacitance parallel with a discrete resistance. $\mathrm{CH} 1$ denotes the input voltage waveform $\left(v_{g}\right)$, while $\mathrm{CH} 2$ represents the output voltage waveform $\left(v_{\mathrm{CH} 2}\right)$. Theory curves were presented based on formula calculation. Measurement data were recorded by channel 1 and channel 2 of the oscilloscope.
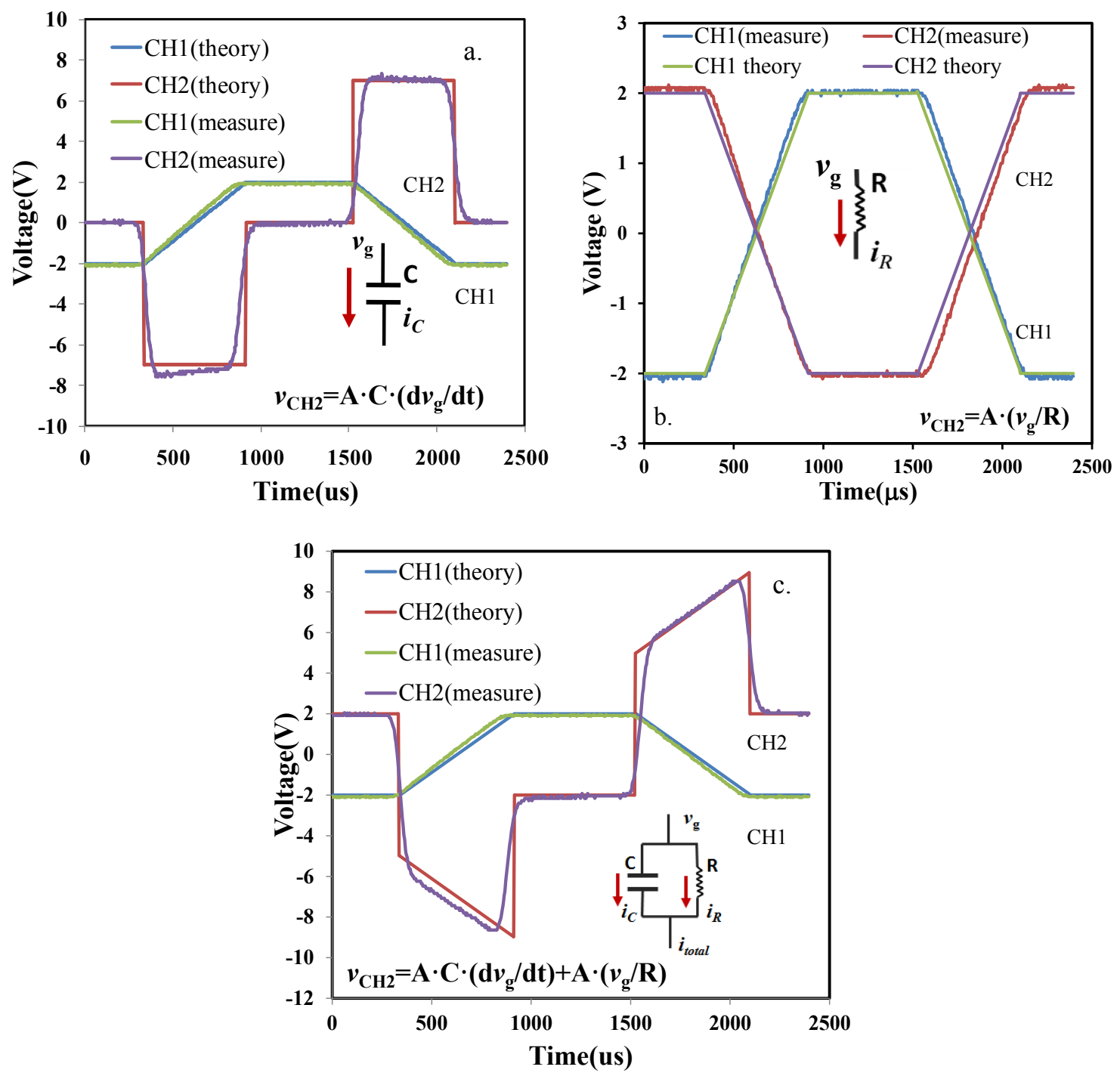
A discrete resistor with a resistance value $R$ as the under testing device to replace the discrete commercial ceramic capacitor was then investigated. The current flowing through the resistor was given:

$$
\begin{gathered}
i_{R}(t)=\frac{v_{\mathrm{CH} 2}(t)}{A}=\frac{v_{g}(t)}{R} \\
v_{\mathrm{CH} 2}(t)=A \cdot \frac{v_{g(t)}}{R}
\end{gathered}
$$

The comparison between theory and measurement is demonstrated in Figure 2b. Like the result of the discrete capacitance, we found the theory and measurement had an impressive consistency.

Finally, the parallel combination of the discrete capacitor and the discrete resistor was merged. The related formula was updated as:

$$
\begin{aligned}
i_{\text {total }}(t)= & \frac{v_{\mathrm{CH} 2}(t)}{A}=i_{C}(t)+i_{R}(t)=C \cdot \frac{d v_{g}(t)}{d t}+\frac{v_{g}(t)}{R} \\
& v_{\mathrm{CH} 2}(t)=A \cdot C \cdot \frac{d v_{g}(t)}{d t}+A \cdot \frac{v_{g}(t)}{R}
\end{aligned}
$$

In Figure 2c, it was found that the theoretical calculation agreed well with measurement data. In summary, the working principle in terms of the pulse CV measurement system is mainly attributed to the current flowing through the device under test. In this paper, the measurement of MOS capacitance with parasitic resistance (usually the value was above $2 \mathrm{M} \Omega$ ) was the key issue.

Before considering a MOS capacitance, the extracted capacitance data from a discrete capacitor should be fully understood. As an example, we tested a $330 \mathrm{pF}$ discrete capacitor. From Equation (2) and an inset in Figure 3, the extracted CV graphs are shown in Figure 3, where we place the original voltage-time (VT) graphs as the inset. Pulse CV up/down (black) means that the CV results were extracted from the rise/fall edge of the input pulse on channel 1 in the inset. Before utilizing the pulse CV technique, a conventional CV test was commonly carried out via Agilent4275/4284 LCR meter (Agilent, Santa Clara, CA, USA). The related CV results (red) are presented in Figure 3. In the conventional test, up/down means the measurement starting from low/high to high/low voltage. The comparison was also made between the conventional test and the pulse CV measurement as shown in Figure 3. Besides high accuracy superposition, the CV curves from the pulse CV measurement need a rise to reach the true capacitance value within the voltage range from -2 to $-1.5 \mathrm{~V}$. Similarly, a fall from the true capacitance value was also observed within the range of $1.5-2 \mathrm{~V}$. The phenomenon was mainly due to the response time of the oscilloscope and the capacitance charging and discharging issues. In consequence, the middle part of the full $\mathrm{CV}$ curves was preferred, which reflected the actual capacitance value of the discrete capacitor, like $-1.5-1.5 \mathrm{~V}$ within the range of $-2-2 \mathrm{~V}$.

After the comparison between the conventional and pulse $\mathrm{CV}$ test for a discrete commercial ceramic capacitor, a thermal oxide $\left(\mathrm{SiO}_{2}\right)$ MOS capacitor sample was investigated. Figure 4a shows the original VT data from both channel one and channel two of the oscilloscope. Calculated from the Equation (6), the related CV results are given in Figure 4b. Similarly, a conventional CV test was employed to compare with the new pulse CV technique. From Figure 4b, vertically the pulse CV and conventional $\mathrm{CV}$ results matched consistently. There was no variation either in accumulation or depletion region. Furthermore, horizontally there was no clear shift for the pulse and conventional CV test. This is because there were no significant traps located in the oxide layer of the thermal $\mathrm{SiO}_{2} \mathrm{MOS}$ system. 
Therefore, it should be the case that no flat-band voltage shift was observed. In other words, the fast (pulse CV) and slow (conventional test) CV measurements were expected to be the same, which was supported by our finding shown in Figure 4b. It can be concluded that the pulse CV system is suitable for MOS capacitance measurement. This provides a convincing basis for investigation of more complicated MOS capacitors with high- $k$ thin films.

Figure 3. Capacitance $(\mathrm{pF})$ versus voltage $(\mathrm{V})$ of a discrete capacitor. Inset: Voltage (V) versus time $(\mu \mathrm{s})$ of a discrete capacitor using the pulse $\mathrm{CV}$ measurement. $\mathrm{CH} 2$ was used to extract the capacitance value. Conventional test up and pulse CV up: $v_{g}$ forward from -2 to $+2 \mathrm{~V}$ (the up trace). Conventional test down and pulse CV down: $v_{g}$ backward from +2 to $-2 \mathrm{~V}$ (the down trace).

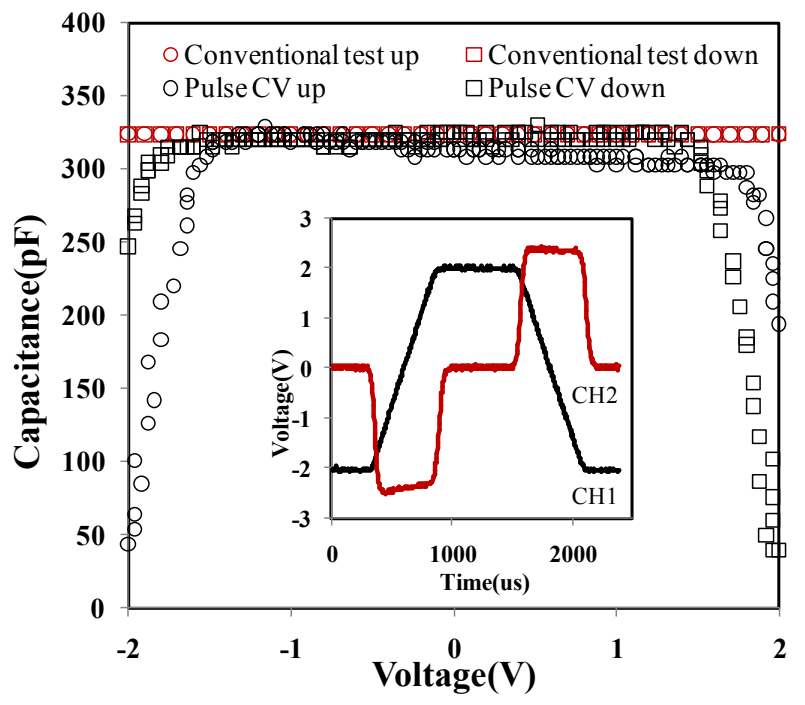

Figure 4. (a) Voltage (V) versus time ( $\mu \mathrm{s})$ of a thermal $\mathrm{SiO}_{2} \mathrm{MOS}$ sample. (b) Capacitance $(\mathrm{pF})$ versus voltage $(\mathrm{V})$ of the $\mathrm{SiO}_{2} \mathrm{MOS}$ sample.
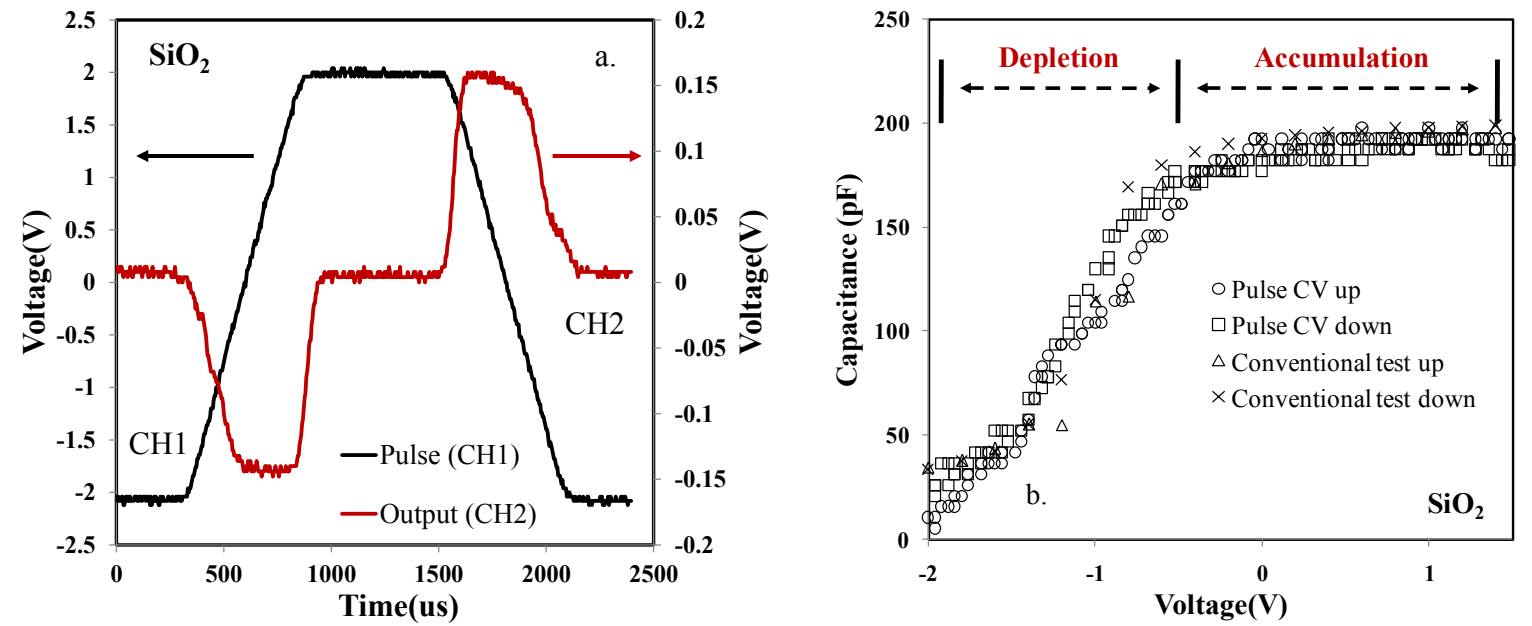

The high- $k$ thin film of $\mathrm{LaAlO}_{3}$ was now under research. The MOS capacitor samples with $\mathrm{LaAlO}_{3}$ were probed using pulse CV technique. The VT results are shown in Figure 5a. Unlike the discrete capacitor and the $\mathrm{SiO}_{2} \mathrm{MOS}$ capacitors introduced before, there were two distinct peaks in channel two in the up (forward from -3 to $+3 \mathrm{~V}$ ) and down (backward from +3 to $-3 \mathrm{~V}$ ) trace of the input pulse, 
respectively. When we did the extraction based on Equation (6) into CV results in Figure 5b, the distinct peaks indicated that a strong inversion occurred when frequency is low. The capacitance density in the accumulation region was $1.27 \times 10^{5} \mathrm{pF} \cdot \mathrm{cm}^{-2}$, when the electric field within the oxide is $7.14 \times 10^{7} \mathrm{~V} \cdot \mathrm{m}^{-1}$. In the inversion region, an inversion layer exists at the silicon surface. In response to the low frequency analog continuous (AC) signal, inversion layer charges can be supplied and removed quickly enough to respond to changes with the gate AC signal voltage, and incremental charge is effectively added or subtracted at the surface of substrate. Most importantly, it was found that a clear horizontal voltage shift happened between the up and down trace. The shift was around 1.3 V. However, only a horizontal voltage shift of $0.4 \mathrm{~V}$ was observed in the same samples from the conventional $\mathrm{CV}$ test. It was proved that the pulse $\mathrm{CV}$ technique was more accurate to track traps in oxides, avoiding trapped electrons/holes recovering/detrapping within the testing time interval of the conventional $\mathrm{CV}$ test. The obvious horizontal voltage shift was induced by trapping and detrapping of electrons/holes between the up and down trace of channel one in Figure $5 \mathrm{a}$. When the positive voltage $(+3 \mathrm{~V})$ is forced on the metal side, net positive charges induce a negative shift of the $\mathrm{C}-\mathrm{V}$ curve. While, if a negative voltage $(-3 \mathrm{~V})$ is applied on the metal side, net negative charges cause a positive shift of the $\mathrm{C}-\mathrm{V}$ curve. As a discussion, a possible explanation is that the shifts might be related to as-grown positive charges and as-grown electron traps. It was reported that there are as-grown fixed positive charges (oxygen vacancies) and as-grown electron traps in high- $k$ oxides [57,73-75]. If both as-grown positive charges and as-grown electron traps in high- $k$ oxides have a high energy level, when $V_{g}<0$, the as-grown positive charges are compensated by electrons, which come from the metal gate and trapped into the as-grown traps; while when $V_{g}>0$, electrons are detrapped to the metal gate through tunneling, which causes that (1) the net charges in the oxide are positive and then (2) the CV curve negatively shifts.

Figure 5. (a) Voltage (V) versus time ( $\mu \mathrm{s})$ of $\mathrm{A} \mathrm{LaAlO}_{3}$ sample. (b) Capacitance (pF) versus voltage $(\mathrm{V})$ of the $\mathrm{LaAlO}_{3}$ sample. In the pulse $\mathrm{CV}$ test, considerable flat-band voltage shift was observed.
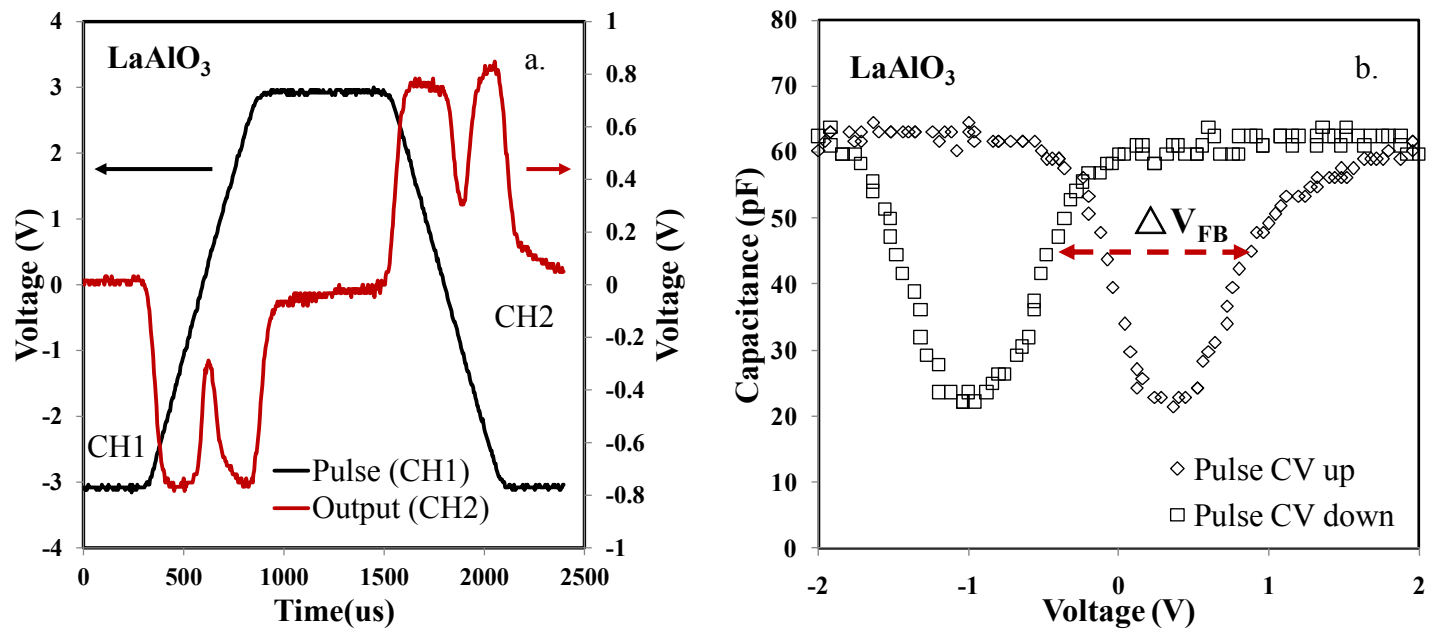

The impact of testing time on the horizontal shift of the CV curve was considered. In the pulse CV technique, edge time for both rise and fall of the voltage pulse was critical to estimate the trap density located in the oxide. The single pulse CV technique described in the paper can be used to accurately measure the charging-induced flat-band voltage shift. Furthermore, a two-pulse CV technique was 
developed to measure the flat-band voltage shift caused by discharging the traps in dielectrics [63]. The rising and falling slopes of a pulse signal applied to the gate will give rise to a displacement current proportional to the capacitance and the pulse ramp rate (see Equation (1)). Less edge time means less test time among each voltage biases. The rise edge time is shown in the inset of Figure 6 . When the edge time was increased to a relatively large value $(\sim 10 \mathrm{~s})$, the test process of the pulse $\mathrm{CV}$ was equal to the conventional CV test carried out via a LCR meter. In Figure 6, it was observed that the flat-band voltage shift was narrowed with increasing edge time and it indicates that the flat-band voltage shift is partially recovered during the edge time of the pulse. Trapped electrons/holes recovering were sensitive to time. Therefore, the time-dependent trapping/detrapping should be probed by short edge time in the pulse CV measurement, which corresponds to shallower traps (traps at a high energy level within oxide), at least within the timescale considered here. While for the larger value of the edge time, the transient shift of the flat-band voltage shift is attributed to slow electron trapping/detrapping. It is also noted that all the ramp-down traces in Figure 6 are almost overlapped, suggesting that the total trapping level changes little during each charging process (positive bias induced). Similar results have been observed and confirmed by using the conventional CV measurement.

Figure 6. Capacitance $(p F)$ versus voltage $(\mathrm{V})$ of a $\mathrm{LaAlO}_{3}$ sample. The variation of flat-band voltage was decreased with increasing pulse edge time.

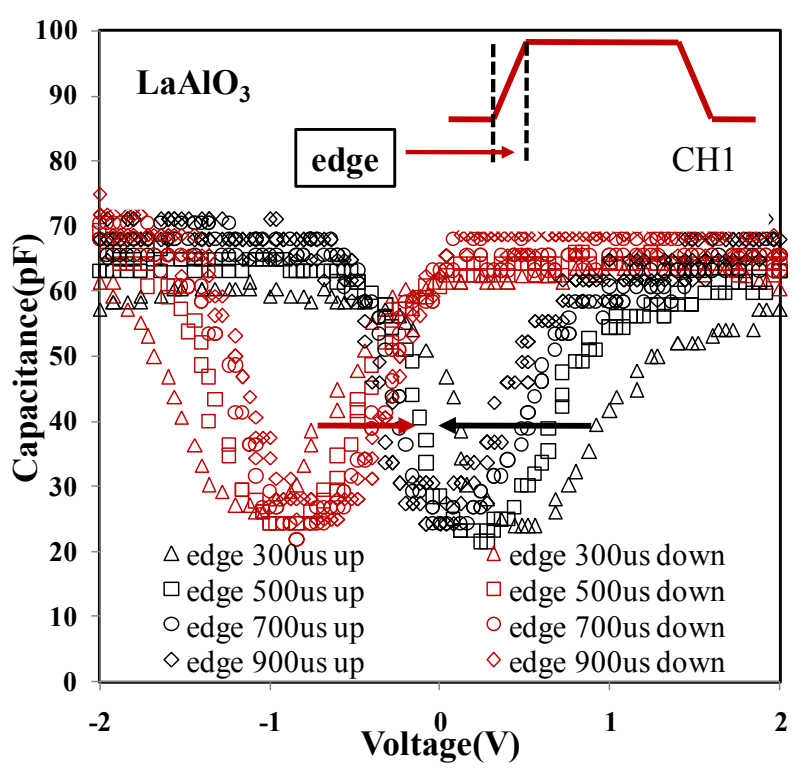

In order to figure out how edge time determines the flat-band voltage shift, two high- $k$ materials $\left(\mathrm{LaAlO}_{3}\right.$ and $\left.\mathrm{NdAlO}_{3}\right)$ plus a thermal oxide were tested, as shown in Figure 7. By adjusting the edge time, the time-dependent trapping/detrapping was tracked correspondingly. Also, it provided a solution to estimate the entire density of net trapped charges within the oxide under specific edge time. For both high- $k$ materials, variations of flat-band voltage were reduced with longer edge time. Due to the hardware limit of the waveform generator, the longest edge time was rated at $900 \mu \mathrm{s}$. When the edge time reached $1 \times 10^{7} \mathrm{~s}(10 \mu \mathrm{s})$, the pulse CV method was supposed to be a type of conventional CV test. The data obtained via a LCR meter are also shown in Figure 7. It was found that for $\mathrm{LaAlO}_{3}$ the variation of the flat-band voltage remained a constant when edge time is less than $400 \mu$ s, which means that the entire density of the net trapped charges within the dielectric was measured if edge time is below $400 \mu \mathrm{s}$. This is 
because, once all as-grown electron traps were filled, $V_{F B}$ would not shift further. The effective trapped charge density now is $1.04 \times 10^{12} \mathrm{~cm}^{-2}\left(=C_{o x} \Delta V_{F B} / q\right.$, where $\Delta V_{F B}=1.31 \mathrm{~V}$ and $C_{o x}=1.27 \times 10^{5} \mathrm{pF} \cdot \mathrm{cm}^{-2}$ is the capacitance density in the accumulation region). $\Delta V_{F B}$ did not return to its fresh value $(0.4 \mathrm{~V})$ even under $900 \mu$ s of edge time, indicating that some electrons are still trapped within the high- $k$ layer. From Figure 7, it was observed that traps of $\mathrm{LaAlO}_{3}$ measured by the conventional $\mathrm{CV}$ method were less than $31 \%$ of the total trapping. Trapping is dominated by the high- $k$ layer, which cannot be probed by charge pumping. The charging within $100 \mathrm{~s}$ is about $30 \%$ of the total, which will be missed if the slow quasi-dc techniques were used. In terms of $\mathrm{NdAlO}_{3}, \Delta V_{F B}$ was recorded at $0.64 \mathrm{~V}$ as the largest value under the edge time of $200 \mu \mathrm{s}$, with trap density of $1.14 \times 10^{12} \mathrm{~cm}^{-2}$ (where $\Delta V_{F B}=0.64 \mathrm{~V}$ and $C_{o x}=2.86 \times 10^{5} \mathrm{pF} \cdot \mathrm{cm}^{-2}$ ). Similar to $\mathrm{LaAlO}_{3}$, the curve trend was also plotted downward and $\Delta V_{F B}$ was close to the fresh value measured using the conventional $\mathrm{CV}$ technique. Within the edge time range of $400-700 \mu \mathrm{s}$, the slope rate of $\mathrm{LaAlO}_{3}$ decreased more significantly than $\mathrm{NdAlO}_{3}$, which indicated that trapped electron detrapping was more sensitive to time. It is noted for $\mathrm{NdAlO}_{3}, \Delta V_{F B}$ did not saturate even at the fastest testing speed of the pulse $(200 \mu \mathrm{s})$. The entire density of net trapped charges shall be captured using a rapider pulse generator and higher measurement resolution. Concerning thermal oxide, there was no flat-band voltage variation under various edge times, which was also supported by the measurement shown in Figure $4 b$.

Figure 7. Variation of flat-band voltage (V) with different edging time ( $\mu$ s) of $\mathrm{NdAlO}_{3}$, $\mathrm{LaAlO}_{3}$ and $\mathrm{SiO}_{2}$ samples.

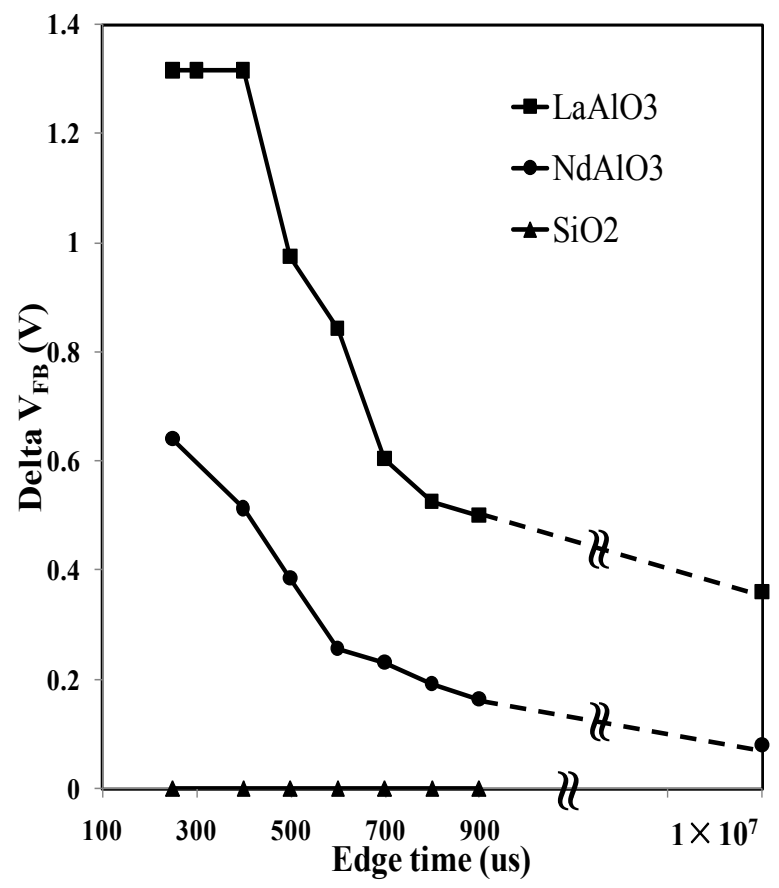

Finally, the variation of $V_{P P}$ was investigated for $\mathrm{LaAlO}_{3}$, where $V_{P P}$ denoted the peak to peak voltage of the pulse. When $V_{P P}$ was $4 \mathrm{~V}$, the pulse started from -2 to $2 \mathrm{~V}$. The definition of $V_{P P}$ could also be referred in the inset of Figure 8a. The relationship between $\triangle V_{F B}$ and edge time under different $V_{P P}$ levels was indicated in Figure 8a. It was observed that with stronger $V_{P P}$ stress, the $\Delta V_{F B}$ remained at a higher level. In all cases, $\triangle V_{F B}$ almost linearly depends on $V_{P P}$ and reaches a value of $1.67 \mathrm{~V}$ at a high voltage $\left(V_{P P}=6 \mathrm{~V}\right)$, denoting a rather strong trapping process in the dielectric stacks. The effective trapped 
charge density is $1.33 \times 10^{12} \mathrm{~cm}^{-2}$ (where $\Delta V_{F B}=1.67 \mathrm{~V}$ and $C_{o x}=1.27 \times 10^{5} \mathrm{pF} \cdot \mathrm{cm}^{-2}$ ), as $V_{P P}$ equals to $6 \mathrm{~V}$ and edge time at $250 \mu$ s. Electron traps with deeper energy levels may exist in the bulk of the high- $k$ layer, which can only be charged with larger gate bias. Slower ramp rates would cause more detrapping during the CV measurements, while higher ramp rates were limited by the trans-amplifier's bandwidth. Figure $8 \mathrm{~b}$ shows the remaining traps during various edge times after taking the pre-existing traps into account. The pre-existing traps are time-independent and also termed as the whole traps. The normalized trapped charge and bar diagram are implemented in Figure $8 \mathrm{~b}$.

In general, following the procedure of the pulse CV test, it would be more convincing to characterize the trapping/detrapping mechanism of the electrons/holes in the high- $k$ materials, which would be used for the development of the next generation non-volatile memory.

Figure 8. (a) Variation of Flat-band Voltage (V) with Different Edging Time ( $\mu \mathrm{s})$ of The $\mathrm{LaAlO}_{3}$, Samples under different VPP level. VPP denotes peak to peak voltage of the pulse. (b) Normalized Trapped Charge with Different Edging Time $(\mu \mathrm{s})$.

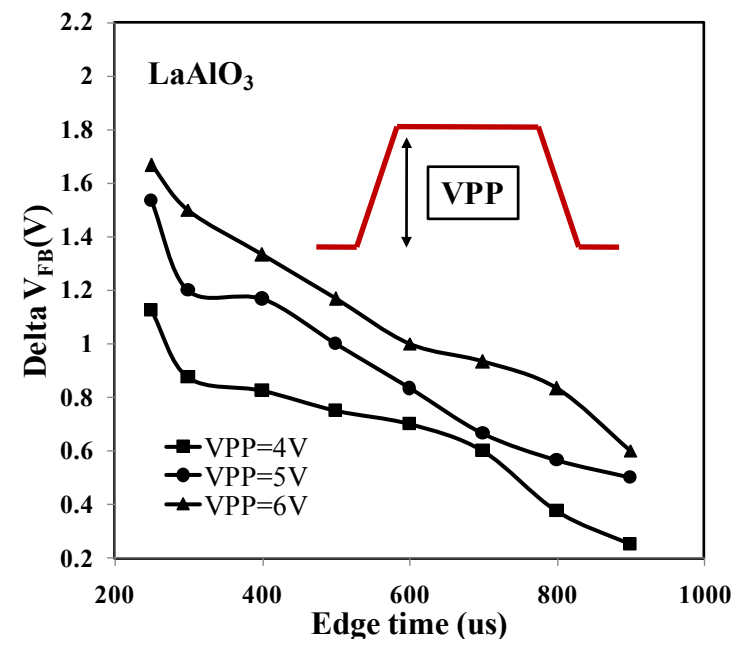

(a)

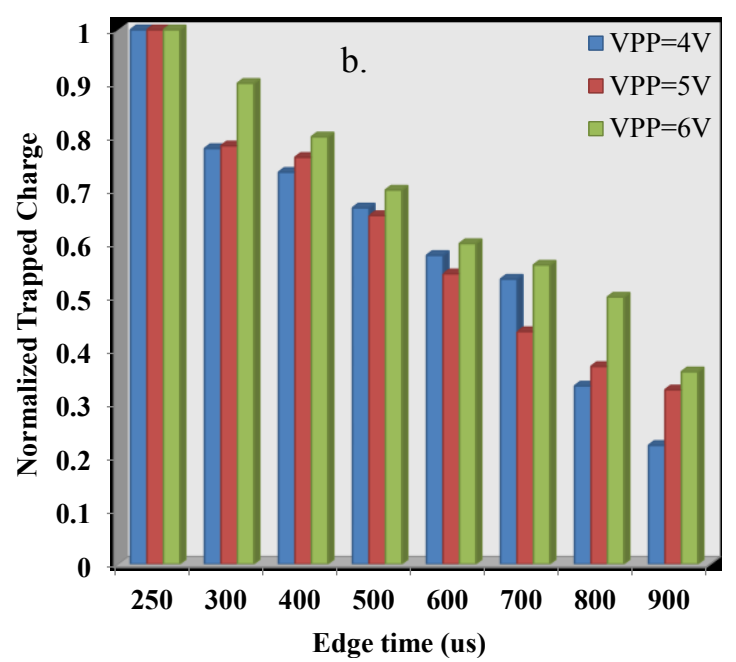

(b)

\section{Conclusions}

In this paper, we have introduced a novel electrical characterization for MOS capacitors with high- $k$ materials: pulse CV measurement. Different from the conventional CV test, the pulse CV technique could complete the whole test within $1 \mathrm{~ms}$. By using the new technique, the testing time (edge time) and the bias/stress time (width time) could be easily adjusted. Compared to thermal oxides, MOS capacitors with high- $k$ materials (like $\mathrm{LaAlO}_{3}$ and $\mathrm{NdAlO}_{3}$ ) showed the intrinsic time-dependent trapping/detrapping mechanism via the pulse CV measurement. Various observations concerning the variation of flat-band voltage were discussed accordingly. After understanding the trapping/detrapping mechanism of the high- $k$ oxides, the pulse $\mathrm{CV}$ technique might be a solid foundation for further exploration into charge-trapping, non-volatile memory based on high- $k$ oxides in the coming future.

\section{Acknowledgments}

This research was funded in part from the Engineering and Physical Science Research Council of UK under the grant EP/D068606/1, the National Natural and Science Foundation of China under the 
grant no. 60976075 and 11375146, the Suzhou Science and Technology Bureau of China under the grant SYG201007 and SYG201223, and the Jiangsu Provincial Science and Technology Supporting Program under the grant BK2012636.

\section{Author Contributions}

Chun Zhao drafted the manuscript. Qifeng Lu and Xiaoyi Yan performed the experiment and extracted the data. Ce Zhou Zhao monitored and led the whole research project. Stephen Taylor and Paul R Chalker participated in the discussions. All of the authors read and approved the final manuscript.

\section{Conflicts of Interest}

The authors declare no conflict of interest.

\section{References}

1. Hoffman, J.; Pan, X.; Reiner, J.W.; Walker, F.; Han, J.P.; Ahn, C.H.; Ma, T.P. Ferroelectric field effect transistors for memory applications. Adv. Mater. 2010, 22, 2957-2961.

2. Wang, M.; He, W.; Ma, T.P. Electron tunneling spectroscopy study of traps in high- $k$ gate dielectrics: Determination of physical locations and energy levels of traps. Appl. Phys. Lett. 2005, 86, doi:10.1063/1.1924893.

3. Zhu, W.J.; Han, J.-P.; Ma, T.P. Mobility measurement and degradation mechanisms of MOSFETs made with ultrathin high- $k$ dielectrics. IEEE Trans. Electron Devices 2004, 51, 98-105.

4. Zhu, W.J.; Ma, T.P.; Tamagawa, T.; Kim, J.; Di, Y. Current transport in metal/hafnium oxide/silicon structure. IEEE Electron Device Lett. 2002, 23, 97-99.

5. Zhao, Y. Design of higher- $k$ and more stable rare earth oxides as gate dielectrics for advanced CMOS devices. Materials 2012, 5, 1413-1438.

6. Tao, J.; Zhao, C.Z.; Zhao, C.; Taechakumput, P.; Werner, M.; Taylor, S.; Chalker, P.R. Extrinsic and intrinsic frequency dispersion of high- $k$ materials in capacitance-voltage measurements. Materials 2012, 5, 1005-1032.

7. Reynolds, G.J.; Kratzer, M.; Dubs, M.; Felzer, H.; Mamazza, R. Electrical properties of thin-film capacitors fabricated using high temperature sputtered modified barium titanate. Materials 2012, 5, 644-660.

8. Suzuki, M. Comprehensive study of lanthanum aluminate high-dielectric-constant gate oxides for advanced CMOS devices. Materials 2012, 5, 443-477.

9. Reynolds, G.J.; Kratzer, M.; Dubs, M.; Felzer, H.; Mamazza, R. Sputtered modified barium titanate for thin-film capacitor applications. Materials 2012, 5, 575-589.

10. Miyata, N. Study of direct-contact $\mathrm{HfO}_{2} / \mathrm{Si}$ interfaces. Materials 2012, 5, 512-527.

11. Kidalov, S.V.; Shakhov, F.M. Thermal conductivity of diamond composites. Materials 2009, 2 , 2467-2495.

12. Zhao, C.; Zhao, C.Z.; Werner, M.; Taylor, S.; Chalker, P.R. Dielectric relaxation of high- $k$ oxides. Nanoscale Res. Lett. 2013, 8, doi:10.1186/1556-276X-8-456. 
13. Zhao, C.; Zhao, C.Z.; Werner, M.; Taylor, S.; Chalker, P.R.; King, P. Grain size dependence of dielectric relaxation in cerium oxide as high- $k$ layer. Nanoscale Res. Lett. 2013, 8, doi:10.1186/ 1556-276X-8-172.

14. Zhao, C.; Zhao, C.Z.; Tao, J.; Werner, M.; Taylor, S.; Chalker, P.R. Dielectric relaxation of lanthanide-based ternary oxides: Physical and mathematical models. J. Nanomater. 2012, 2012, doi:10.1155/2012/241470.

15. Zhao, C.; Zhao, C.Z.; Werner, M.; Taylor, S.; Chalker, P.R. Advanced CMOS gate stack: Present research progress. ISRN Nanotechnol. 2012, 2012, doi:10.5402/2012/689023.

16. Zhao, C.Z.; Werner, M.; Taylor, S.; Chalker, P.R.; Jones, A.C.; Zhao, C. Dielectric relaxation of la-doped zirconia caused by annealing ambient. Nanoscale Res. Lett. 2011, 6, doi:10.1007/ s11671-010-9782-z.

17. Taechakumput, P.; Zhao, C.Z.; Taylor, S.; Werner, M.; Chalker, P.R.; Gaskell, J.M.; Aspinall, H.C.; Jones, A.C.; Chen, S.S. Thermal stability of neodymium aluminates high- $k$ dielectric deposited by liquid injection MOCVD using single-source heterometallic alkoxide precursors. J. Nanomater. 2012, 2012, doi:10.1155/2012/891079.

18. Zhao, C.Z.; Taylor, S.; Werner, M.; Chalker, P.R.; Murray, R.T.; Gaskell, J.M.; Jones, A.C. Dielectric relaxation of lanthanum doped zirconium oxide. J. Appl. Phys. 2009, 105, doi:10.1063/1.3078038.

19. Zhao, C.Z.; Taylor, S.; Werner, M.; Chalker, P.R.; Gaskell, J.M.; Jones, A.C. Frequency dispersion and dielectric relaxation of $\mathrm{La}_{2} \mathrm{Hf}_{2} \mathrm{O}_{7}$. J. Vac. Sci. Technol. B 2009, 27, 333-337.

20. Zhao, C.Z.; Taylor, S.; Werner, M.; Chalker, P.R.; Potter, R.J.; Gaskell, J.M.; Jones, A.C. High- $k$ materials and their response to gamma ray radiation. J. Vac. Sci. Technol. B Microelectron. Nanometer Struct. 2009, 27, 411-415.

21. Werner, M.; King, P.J.; Hindley, S.; Romani, S.; Mather, S.; Chalker, P.R.; Williams, P.A.; van den Berg, J.A. Atomic layer deposition of $\mathrm{Ti}^{-}-\mathrm{HfO}_{2}$ dielectrics. J. Vac. Sci. Technol. A 2013, 31, doi:10.1116/1.4748570.

22. Mather, S.; Sedghi, N.; Althobaiti, M.; Mitrovic, I.Z.; Dhanak, V.; Chalker, P.R.; Hall, S. Low EOT $\mathrm{GeO}_{2} / \mathrm{Al}_{2} \mathrm{O}_{3} / \mathrm{HfO}_{2}$ on $\mathrm{Ge}$ substrate using ultrathin $\mathrm{Al}$ deposition. Microelectron. Eng. 2013, 109, 126-128.

23. King, P.J.; Werner, M.; Chalker, P.R.; Jones, A.C.; Aspinall, H.C.; Basca, J.; Wrench, J.S.; Black, K.; Davies, H.O.; Heys, P.N. Effect of deposition temperature on the properties of $\mathrm{CeO}_{2}$ films grown by atomic layer deposition. Thin Solid Films 2011, 519, 4192-4195.

24. Chalker, P.R.; Marshall, P.A.; King, P.J.; Dawson, K.; Romani, R.; Williams, P.A.; Ridealgh, J.; Rosseinsky, M.J. Atomic layer deposition of germanium-doped zinc oxide films with tuneable ultraviolet emission. J. Mater. Chem. 2012, 22, 12824-12829.

25. Yan, L.; Xu, Z.L.; Grygiel, C.; McMitchell, S.R.C.; Suchomel, M.R.; Bacsa, J.; Clark, J.H.; Niu, H.J.; Romani, S.; Palgrave, R.G.; et al. $\operatorname{SrHf}(0.67) \mathrm{Ti}(0.33) \mathrm{O}(3)$ high- $k$ films deposited on Si by pulsed laser deposition. Appl. Phys. A Mater. Sci. Process. 2011, 104, 447-451.

26. Moram, M.A.; Zhang, Y.; Joyce, T.B.; Holec, D.; Chalker, P.R.; Mayrhofer, P.H.; Kappers, M.J.; Humphreys, C.J. Structural properties of wurtzitelike $\mathrm{ScGaN}$ films grown by $\mathrm{NH}_{3}$-molecular beam epitaxy. J. Appl. Phys. 2009, 106, 113533:1-113533:6. 
27. Aspinall, H.C.; Bacsa, J.; Jones, A.C.; Wrench, J.S.; Black, K.; Chalker, P.R.; King, P.J.; Marshall, P.; Werner, M.; Davies, H.O.; Odedra, R. Ce(IV) complexes with donor-functionalized alkoxide ligands: Improved precursors for chemical vapor deposition of $\mathrm{CeO}_{2}$. Inorg. Chem. 2011, 50, 11644-11652.

28. Gross, M.S.; Ulla, M.A.; Querini, C.A. Catalytic oxidation of diesel soot: New characterization and kinetic evidence related to the reaction mechanism on $\mathrm{K} / \mathrm{CeO}_{2}$ catalyst. Appl. Catal. A 2009, 360, 81-88.

29. Pan, T.M.; Liao, C.S.; Hsu, H.H.; Chen, C.L.; Lee, J.D.; Wang, K.T.; Wang, J.C. Excellent frequency dispersion of thin gadolinium oxide high- $k$ gate dielectrics. Appl. Phys. Lett. 2005, 87, 262908:1-262908:3.

30. Liu, C.H.; Pan, T.M.; Shu, W.H.; Huang, K.C. Physical and electrical properties of Ti-doped $\mathrm{Er}_{2} \mathrm{O}_{3}$ films for high- $k$ gate dielectrics. Electrochem. Solid-State Lett. 2007, 10, 54-57.

31. Anthony, J.; Aspinall, H.C.; Chalker, P.R.; Potter, R.J.; Manning, T.D.; Loo, Y.F.; O'Kane, R.; Gaskell, J.M.; Smith, L.M. MOCVD and ALD of high- $k$ dielectric oxides using Alkoxide precursors. Chem. Vapor Depos. 2006, 12, 83-98.

32. Adamopoulos, G.; Thomas, S.; Bradley, D.D.; McLachlan, M.A.; Anthopoulos, T.D. Low-voltage $\mathrm{ZnO}$ thin-film transistors based on $\mathrm{Y}_{2} \mathrm{O}_{3}$ and $\mathrm{Al}_{2} \mathrm{O}_{3}$ high- $k$ dielectrics deposited by spray pyrolysis in air. Appl. Phys. Lett. 2011, 98, 123503:1-123503:3.

33. Lu, X.B.; Liu, Z.G.; Zhang, X.; Huang, R.; Zhou, H.W.; Wang, X.P.; Nguyen, B.Y. Investigation of high-quality ultra-thin $\mathrm{LaAlO}_{3}$ films as high- $k$ gate dielectrics. J. Phys. D Appl. Phys. 2003, 36, doi:10.1088/0022-3727/36/23/027.

34. Gougousi, T.; Kelly, M.J.; Terry, D.B.; Parsons, G.N. Properties of La-silicate high- $k$ dielectric films formed by oxidation of La on silicon. J. Appl. Phys. 2003, 93, 1691-1696.

35. Mahata, C.M.; Bera, K.; Das, T.; Mallik, S.; Hota, M.K.; Majhi, B.; Verma, S.; Bose, P.K.; Maiti, C.K. Charge trapping and reliability characteristics of sputtered $\mathrm{Y}_{2} \mathrm{O}_{3}$ high- $k$ dielectrics on N- and S-passivated germanium. Semicond. Sci. Technol. 2009, 24, doi:10.1088/0268-1242/ 24/8/085006.

36. Pan, T.M.; Lei, T.F.; Chao, T.S.; Chang, K.L.; Hsieh, K.C. High quality ultrathin $\mathrm{CoTiO}_{3}$ high- $k$ gate dielectrics. Electrochem. Solid-State Lett. 2000, 3, 433-434.

37. Kim, S.K.; Kim, K.M.; Kwon, O.S.; Lee, S.W.; Jeon, C.B.; Park, W.Y.; Hwang, C.S.; Jeong, J. Structurally and electrically uniform deposition of high- $k \mathrm{TiO}_{2}$ thin films on a $\mathrm{Ru}$ electrode in three-dimensional contact holes using atomic layer deposition. Electrochem. Solid-State Lett. 2005, 8, F59-F62.

38. Adamopoulos, G.; Thomas, S.; Wöbkenberg, P.H.; Bradley, D.D.; McLachlan, M.A.; Anthopoulos, T.D. High-mobility low-voltage $\mathrm{ZnO}$ and Li-doped $\mathrm{ZnO}$ transistors based on $\mathrm{ZrO}_{2}$ high- $k$ dielectric grown by spray pyrolysis in ambient air. Adv. Mater. 2011, 23, 1894-1898.

39. Gaskell, J.M.; Jones, A.C.; Chalker, P.R.; Werner, M.; Aspinall, H.C.; Taylor, S.; Taechakumput, P.; Heys, P.N. Deposition of lanthanum zirconium oxide high- $k$ films by liquid injection ALD and MOCVD. Chem. Vapor Depos. 2007, 13, 684-690.

40. Gutowski, M.; Jaffe, J.E.; Liu, C.L.; Stoker, M.; Hegde, R.I.; Rai, R.S.; Tobin, P.J. Thermodynamic stability of high- $k$ dielectric metal oxides $\mathrm{ZrO}_{2}$ and $\mathrm{HfO}_{2}$ in contact with $\mathrm{Si}$ and $\mathrm{SiO}_{2}$. Appl. Phys. Lett. 2002, 80, 1897-1899. 
41. Dimoulas, A.; Vellianitis, G.; Mavrou, G.; Apostolopoulos, G.; Travlos, A.; Wiemer, C.; Fanciulli, M.; Rittersma, Z.M. $\mathrm{La}_{2} \mathrm{Hf}_{2} \mathrm{O}_{7}$ high- $k$ gate dielectric grown directly on $\mathrm{Si}(001)$ by molecular-beam epitaxy. Appl. Phys. Lett. 2004, 85, 3205-3207.

42. Gang, H.; Deng, B.; Sun, Z.Q.; Chen, X.S.; Liu, Y.M.; Zhang, L.D. CVD-derived Hf-based High- $k$ Gate Dielectrics. Crit. Rev. Solid State Mater. Sci. 2013, 38, 235-261.

43. Watanabe, H.; Saitoh, M.; Ikarashi, N.; Tatsumi, T. High-quality HfSixOy gate dielectrics fabricated by solid phase interface reaction between physical-vapor-deposited metal-Hf and $\mathrm{SiO}_{2}$ underlayer. Appl. Phys. Lett. 2004, 85, 449-451.

44. Darbandy, G.; Ritzenthaler, R.; Lime, F.; Garduño, I.; Estrada, M.; Cerdeira, A.; Iñiguez, B. Analytical modeling of direct tunneling current through gate stacks for the determination of suitable high- $k$ dielectrics for nanoscale double-gate MOSFETs. Semicond. Sci. Technol. 2011, 26, doi:10.1088/0268-1242/26/4/045002.

45. Myllymäki, P.; Roeckerath, M.; Putkonen, M.; Lenk, S.; Schubert, J.; Niinistö, L.; Mantl, S. Characterization and electrical properties of high- $k \mathrm{GdScO}_{3}$ thin films grown by atomic layer deposition. Appl. Phys. A 2007, 88, 633-637.

46. Chan, K.C.; Lee, P.F.; Li, D.F.; Dai, J.Y. Memory characteristics and the tunneling mechanism of Au nanocrystals embedded in a $\mathrm{DyScO}_{3}$ high- $k$ gate dielectric layer. Semicond. Sci. Technol. 2011, 26, doi:10.1088/0268-1242/26/2/025015.

47. Milanov, A.P.; Xu, K.; Cwik, S.; Parala, H.; de los Arcos, T.; Becker, H.W.; Devi, A. $\mathrm{Sc}_{2} \mathrm{O}_{3}, \mathrm{Er}_{2} \mathrm{O}_{3}$, and $\mathrm{Y}_{2} \mathrm{O}_{3}$ thin films by MOCVD from volatile guanidinate class of rare-earth precursors. Dalton Trans. 2012, 41, 13936-13947.

48. Gaskell, J.M.; Jones, A.C.; Aspinall, H.C.; Przybylak, S.; Chalker, P.R.; Black, K.; Davies, H.O.; Taechakumput, P.; Taylor, S.; Critchlow, G.W. Liquid injection ALD and MOCVD of lanthanum aluminate using a bimetallic alkoxide precursor. J. Mater. Chem. 2006, 16, 3854-3860.

49. Gaskell, J.M.; Przybylak, S.; Jones, A.C.; Aspinall, H.C.; Chalker, P.R.; Black, K.; Potter, R.J.; Taechakumput, P.; Taylor, S. Deposition of Pr- and Nd-aluminate by liquid injection MOCVD and ALD using single-source heterometallic alkoxide precursors. Chem. Mater. 2007, 19, 4796-4803.

50. Ma, T.P.; Han, J.P. Why is nonvolatile ferroelectric memory field-effect transistor still elusive? IEEE Electron Device Lett. 2002, 23, 386-388.

51. She, M.; King, T.J.; Hu, C.M.; Zhu, W.J.; Luo, Z.J.; Han, J.P.; Ma, T.P. JVD silicon nitride as tunnel dielectric in p-channel flash memory. IEEE Electron Device Lett. 2002, 23, 91-93.

52. Lee, C.H.; Hur, S.H.; Shin, Y.C.; Choi, J.H.; Park, D.G.; Kim, K. Charge-trapping device structure of dielectric for high-density flash memory. Appl. Phys. Lett. 2005, 86, doi:10.1063/1.1897431.

53. Lee, C.H.; Park, K.C.; Kim, K. Charge-trapping memory cell of $\mathrm{SiO}_{2} / \mathrm{SiN} /$ high- $k$ dielectric $\mathrm{Al}_{2} \mathrm{O}_{3}$ with TaN metal gate for suppressing backward-tunneling effect. Appl. Phys. Lett. 2005, 87, 073510:1-073510:3.

54. Zhang, G.; Wang, L.K.; Won, J.Y.; Li, M.F. Spatial distribution of charge traps in a SONOS-type Flash memory using a high- $k$ trapping layer. IEEE Trans. Electron Devices 2007, 54, 3317-3324.

55. You, H.W.; Cho, W.J. Charge trapping properties of the $\mathrm{HfO}_{2}$ layer with various thicknesses for charge trap flash memory applications. Appl. Phys. Lett. 2009, 96, 093506:1-093506:3.

56. Lu, C.Y.; Hsieh, K.Y.; Liu, R. Future challenges of flash memory technologies. Microelectron. Eng. 2009, 86, 283-286. 
57. Zhao, C.Z.; Zhang, J.F.; Groeseneken, G.; Degraeve, R. Hole-traps in silicon dioxides-Part II: Generation mechanism. IEEE Trans. Electron Devices 2004, 51, 1274-1280.

58. Zhao, C.Z.; Zhang, J.F. Effects of hydrogen on positive charges in gate oxides. J. Appl. Phys. 2005, 97, doi:10.1063/1.1882771.

59. Zhao, C.Z.; Zhang, J.F.; Zahid, M.B.; Groeseneken, G.; Degraeve, R.; de Gendt, S. Impact of gate materials on positive charge formation in $\mathrm{HfO}_{2} / \mathrm{SiO}_{2}$ stacks. Appl. Phys. Lett. 2006, 89, doi:10.1063/1.2220484.

60. Young, C.D.; Zeitzoff, P.; Brown, A.; Bersuker, G.; Lee, B.H.; Hauser, J.R. Intrinsic mobility evaluation of high- $k$ gate dielectric transistors using pulsed $\mathrm{I}_{\mathrm{d}}-\mathrm{V}_{\mathrm{g}}$. IEEE Electron Device Lett. 2005, 26, 586-589.

61. Bersuker, G.; Zeitzoff, P.; Sim, J.H.; Lee, B.H.; Choi, R.; Brown, G.; Young, C.D. Mobility evaluation in transistors with charge-trapping gate dielectrics. Appl. Phys. Lett. 2005, 87, doi:10.1063/1.1995956.

62. Puzzilli, G.; Govoreanu, B.; Irrera, F.; Rosmeulenb, M.; van Houdt, J. Characterization of charge trapping in $\mathrm{SiO}_{2} / \mathrm{Al}_{2} \mathrm{O}_{3}$ dielectric stacks by pulsed $\mathrm{C}-\mathrm{V}$ technique. Microelectron. Reliab. 2007, 47, $508-512$.

63. Zhang, W.D.; Govoreanu, B.; Zheng, X.F.; Aguado, D.R.; Rosmeulen, M.; Blomme, P.; Zhang, J.F.; van Houdt, J. Two-pulse C-V: A new method for characterizing electron traps in the bulk of $\mathrm{SiO}_{2} /$ high- $k$ dielectric stacks. IEEE Electron Device Lett. 2008, 29, 1043-1046.

64. Zheng, X.F.; Zhang, W.D.; Govoreanu, B.; Aguado, D.R.; Zhang, J.F.; van Houdt, J. Energy and spatial distributions of electron traps throughout $\mathrm{SiO}_{2} / \mathrm{Al}_{2} \mathrm{O}_{3}$ stacks as the IPD in flash memory application. IEEE Trans. Electron Devices 2010, 57, 288-296.

65. Zheng, X.F.; Zhang, W.D.; Govoreanu, B.; Zhang, J.F.; van Houdt, J. A new multi-pulse technique for probing electron trap energy distribution in high-kappa materials for Flash memory application. IEEE Trans. Electron Devices 2010, 57, 2484-2492.

66. ManjulaRani, K.N.; Ramgopal Rao, V.; Vasi, J. A new method to characterize border traps in submicron transistors using hysteresis in the drain current. IEEE Trans. Electron Devices 2003, 50, 973-979.

67. Degraeve, R.; Cho, M.; Govoreanu, B.; Kaczer, B.; Zahid, M.B.; van Houdt, J.; Jurczak, M.; Groeseneken, G. Trap spectroscopy by charge injection and sensing (TSCIS): A quantitative electrical technique for studying defects in dielectric stacks. In Proceedings of the IEEE International Electron Devices Meeting (IEDM), San Francisco, CA, USA, 15-17 December 2008.

68. Sun, X.; Xu, N.; Xue, F.; Alian, A.; Andrieu, F.; Nguyen, B.Y.; Poiroux, T.; Faynot, O.; Lee, J.; Cui, S.; Ma, T.P. AC transconductance: A novel method to characterize oxide traps in advanced FETs without a body contact. In Proceedings of the IEEE International Electron Devices Meeting IEDM, San Francisco, CA, USA, 10-13 December 2012.

69. Zheng, X.F.; Robinson, C.; Zhang, W.D.; Zhang, J.F.; Govoreanu, B.; van Houdt, J. Electron trapping in HfAlO high-kappa stack for Flash memory applications: An origin of $\mathrm{V}_{\text {th }}$ window closure during cycling operations. IEEE Trans. Electron Devices 2011, 58, 1344-1351.

70. Benbakhti, B.; Zhang, J.F.; Ji, Z.; Zhang, W.; Mitard, J.; Kaczer, B.; Groeseneken, G.; Hall, S.; Chalker, P.; Robertson, J. Characterization of electron traps in Si-capped Ge MOSFETs with $\mathrm{HfO}_{2} / \mathrm{SiO}_{2}$ gate stack. IEEE Electron Device Lett. 2012, 33, 1681-1683. 
71. Duan, M.; Zhang, J.F.; Ji, Z.; Zhang, W.; Kaczer, B.; de Gendt, S.; Groeseneken, G. Defect loss: A new concept for reliability of MOSFETs. IEEE Electron Device Lett. 2012, 33, 480-482.

72. Tang, B.; Robinson, C.; Zhang, W.D.; Zhang, J.F.; Degraeve, R.; Blomme, P.; Toledano-Luque, M.; van den Bosch, G.; Govoreanu, B.; van Houdt, J. Read and pass disturbance in the programmed states of floating gate flash memory cells with high- $k$ interpoly gate dielectric stacks. IEEE Trans. Electron Devices 2013, 60, 2261-2267.

73. Zhao, C.Z.; Zahid, M.B.; Zhang, J.F.; Groeseneken, G.; Degraeve, R.; de Gendt, S. Threshold voltage instability of $p$-channel metal-oxide-semiconductor field effect transistors with hafnium based dielectrics. Appl. Phys. Lett. 2007, 90, 143502:1-143502:3.

74. Zhang, J.F.; Zhao, C.Z.; Zahid, M.B.; Groeseneken, G.; Degraeve, R.; de Gendt, S. An assessment of the location of as-grown electron traps in $\mathrm{HfO}_{2} / \mathrm{HfSiO}$ stacks. IEEE Electron Device Lett. 2006, 27, 817-820.

75. Zhao, C.Z.; Zhang, J.F.; Chang, M.H.; Peaker, A.R.; Hall, S.; Groeseneken, G.; Pantisano, L.; de Gendt, S.; Heyns, M. Stress-induced positive charge in hf-based gate dielectrics: Impact on device performance and a framework for the defect. IEEE Trans. Electron Device 2008, 55, $1647-1655$.

(C) 2014 by the authors; licensee MDPI, Basel, Switzerland. This article is an open access article distributed under the terms and conditions of the Creative Commons Attribution license (http://creativecommons.org/licenses/by/4.0/). 